\title{
Accelerated Dose Escalation with Three Injections of an Aluminum Hydroxide-Adsorbed Allergoid Preparation of Six Grasses Is Safe for Patients with Moderate to Severe Allergic Rhinitis
}

\author{
Matthias Volkmar Kopp ${ }^{a, b} \quad$ Xenia Bovermann ${ }^{a, b} \quad$ Ludger Klimek $^{c}$ \\ ${ }^{a}$ Department of Pediatric Pulmonology and Allergology, University of Lübeck, University Medical Center \\ Schleswig-Holstein, Lübeck, Germany; ${ }^{b}$ Airway Research Center North, Member of the German Center of Lung \\ Research (DZL), Lübeck, Germany; ' Zentrum für Rhinologie und Allergologie, Wiesbaden, Germany
}

\section{Keywords}

Allergic rhinitis · Allergen-specific immunotherapy ·

Allergoids · Accelerated dose escalation · Side effects

\begin{abstract}
Only few data on safety during high-dose, accelerated escalation schedules during subcutaneous allergen immunotherapy (AIT) are available. The aim of this study was to assess the safety and tolerability of an accelerated dose escalation schedule of AIT in adult patients with moderate to severe seasonal rhinoconjunctivitis in a multicenter, openlabel, randomized phase II trial. The dose escalation scheme for patients in Group I ( 1 strength) included 3 injections with 1 strength, B $(10,000 \mathrm{TU} / \mathrm{mL})$, whereas the dose escalation scheme for Group II (standard) included 7 injections with 2 strengths, $A(1,000 \mathrm{TU} / \mathrm{mL})$ and $B(10,000 \mathrm{TU} / \mathrm{mL})$, of an aluminum hydroxide-adsorbed allergoid grass pollen preparation. Overall, 72 of 87 randomized patients (83.7\%) reported at least 1 treatment-emergent adverse event (TEAE; 82.2 [Group I] vs. $85.4 \%$ [Group II]); $58.8 \%$ of all reported TEAEs were assessed as being related to AIT (60.0 vs. $48.8 \%$ ). The most frequently reported AIT-related TEAEs were swelling (46.7 vs. $34.1 \%$ ), erythema (28.9 vs. $36.6 \%$ ), and pruritus (31.1 vs. $17.1 \%)$ at the site of the injection. Systemic allergic reac-
\end{abstract}

\begin{tabular}{ll}
\hline KARGER & ( 2019 The Author(s) \\
& Published by S. Karger AG, Basel Oparger \\
E-Mail karger@karger.com & This article is licensed under the Creative Commons Attribution- \\
www.karger.com/iaa & NonCommercial-NoDerivatives 4.0 International License (CC BY- \\
NC-ND) (http://www.karger.com/Services/OpenAccessLicense). \\
Usage and distribution for commercial purposes as well as any dis- \\
tribution of modified material requires written permission.
\end{tabular}

tions occurred in 5 (5.8\%) patients overall, with more being reported in the 1-strength group (4 [8.9\%] vs. 1 [2.4\%]). All systemic allergic reactions were classified as World Allergy Organization (WAO) Grade 1 or Grade 2 reactions. Accelerated high-dose escalation with an aluminum hydroxide-adsorbed grass pollen allergoid can be initiated with a safety and tolerability profile comparable to the standard dose escalation schedule in patients with allergic rhinitis with or without asthma.

(C) 2019 The Author(s)

Published by S. Karger AG, Basel

\section{Introduction}

Allergic rhinitis (AR), although recognized as the most common form of allergy, is often underdiagnosed and insufficiently treated. The prevalence of AR is estimated to be as high as $10-30 \%$ of the population, with the greatest frequency found in children and adolescents [1]. Moreover, AR is a systemic allergic disease with numerous comorbid disorders, including asthma, eczema, food allergies, eosinophilic esophagitis (EoE), olfaction disorders, obstructive sleep apnea, and other symptoms [2].

Edited by: H.-U. Simon, Bern.
Dr. Matthias Volkmar Kopp

Department of Pediatric Pulmonology and Allergy

University of Lübeck, Ratzeburger Allee 160

DE-23538 Lübeck (Germany)

E-Mail matthias.kopp@uksh.de 
Allergen-specific immunotherapy is highly effective in patients with AR and allergic asthma [3]; up to now, it is the only treatment that can modify the underlying course of allergic diseases [4]. One hallmark of subcutaneous immunotherapy (SCIT) is that increasing allergen doses are applied to reach the efficacious maintenance phase. However, only few data on the safety and tolerability of different protocols of accelerated dose escalation are available. The efficacy of SCIT with grass pollen allergoids has been demonstrated in previous trials.

Corrigan et al. [5] demonstrated significant differences of 26.6 and $48.4 \%$ in symptom and medication score in the first and second year between active and placebo treatment, respectively, in favor of the grass pollen allergoid ( $p=0.028$ and 0.018). Recently, Chaker et al. [6] studied an accelerated dose escalation schedule with a grass pollen allergoid in 122 adult patients with AR with or without asthma. They demonstrated that the accelerated 4-dose escalation scheme (starting with 200 therapeutic units [TU]) can be administered, with a favorable safety profile comparable to the standard 7-dose escalation regime that starts with 100 TU. Of note, there were only mild systemic reactions in both groups with symptoms graded as World Allergy Organization (WAO) Grade 1 or 2.

Here, we aimed to go 1 step further, starting with an acceleration scheme of 3 injections and an initial dose of $1,000 \mathrm{TU}$. We hypothesize that a further acceleration of dose escalation is possible, with a favorable safety profile and good tolerability. Moreover, accelerated dose schedules might offer the opportunity to gain clinical and immunological tolerance faster than with standard dose escalation. In the long run, a reduction in the number of doctor's visits and/or an improved tolerance might increase compliance and adherence to SCIT in our patients. Therefore, the main objective of this therapeutic phase II trial was to evaluate the safety and tolerability of an accelerated high-dose escalation schedule (1 strength) of specific immunotherapy with a grass pollen allergoid for adult patients with AR or rhinoconjunctivitis (with or without allergic asthma) caused by grass pollen, and compare it to the standard specific immunotherapy with a 2-strength grass pollen allergoid.

\section{Material and Methods}

\section{Trial Design and Population}

This was a multicenter, randomized, open-label, phase II clinical trial in adult patients with moderate to severe AR or rhinoconjunctivitis. It was conducted in Germany, Poland, Spain, and Rus- sia. To be eligible for trial participation, patients had to meet the following inclusion criteria: an age between 18 and $\leq 65$ years; IgEmediated seasonal AR or rhinoconjunctivitis with or without allergic asthma, a positive skin prick test ( $\geq 3 \mathrm{~mm}$ in diameter) and specific IgE ( $\geq 0.70 \mathrm{kU} / \mathrm{L})$ against grass pollen; at least 1 month of AR or rhinoconjunctivitis symptoms triggered by grass pollen exposure in the period May to August; previous antiallergic treatment for at least 2 seasons prior to enrollment; and written informed consent. In cases with a diagnosis of asthma, the asthma had to be classified as "well controlled" according to the GINA guidelines [7]. Exclusion criteria were: a history of confirmed anaphylaxis after an AIT injection; AIT with grass pollen within the last 5 years; current treatment with any kind of immunotherapy; uncontrolled/partly controlled asthma according to the GINA guidelines; autoimmune diseases; $\beta$-blocker use; and a contraindication for the use of adrenalin.

Patients were randomized into an accelerated dose escalation group (Group I or "1-strength group") and a standard dose escalation group (Group II or "standard group"). The trial was conducted out of season in autumn and winter, i.e., prior to the grass pollen season. All patients were recruited between October and March 2018 (the first patient on 4 October 2017 and the last patient on 31 May 2018). No placebo group was included in this trial. EudraCT 2017-000754-19.

\section{Test Product and Treatment}

The grass pollen allergoid (Allergopharma $\mathrm{GmbH}$ and Co., KG, Reinbek, Germany) was a mixture of allergens from 6 grass pollen species (Holcus lanatus, Dactylis glomerata, Lolium perenne, Phleum pratense, Poa pratensis, and Festuca pratensis) which are chemically modified with formaldehyde. The allergoid is coprecipitated with aluminium hydroxide. The preparation is provided in 2 strengths: $\mathrm{A}(1,000 \mathrm{TU} / \mathrm{mL})$ and $\mathrm{B}(10,000 \mathrm{TU} / \mathrm{mL})$.

The 1-strength group received only 3 injections of 1 strength (B) of the grass pollen allergoid $(0.1 \mathrm{~mL}$ of $1,000 \mathrm{TU}, 0.3 \mathrm{~mL}$ of 3,000 $\mathrm{TU}$, and $0.6 \mathrm{~mL}$ of $6,000 \mathrm{TU})$ at a weekly interval. The standard group started with $1 / 10$ of the dose of the 1-strength group and received 7 injections (strength A: $0.1 \mathrm{~mL}$ of $100 \mathrm{TU}, 0.2 \mathrm{~mL}$ of 200 TU, $0.4 \mathrm{~mL}$ of $400 \mathrm{TU}$, and $0.8 \mathrm{~mL}$ of $800 \mathrm{TU}$; strength $\mathrm{B}: 0.15 \mathrm{~mL}$ of $1,500 \mathrm{TU}, 0.3 \mathrm{~mL}$ of $3,000 \mathrm{TU}$, and $0.6 \mathrm{~mL}$ of $6,000 \mathrm{TU})$. When the maintenance dose had been reached, both groups received 2 maximum-dose injections $(0.6 \mathrm{~mL}$ of $6,000 \mathrm{TU})$ of strength $\mathrm{B}$ after 14 and 28 days. After each SCIT injection, patients in both groups were supervised for at least $30 \mathrm{~min}$. Dosage modification was performed if local and/or systemic adverse events (AEs) occurred, and this was based on a predefined regime.

\section{Assessment of AR and Asthma}

To characterize AR severity, the investigator had to judge the severity grade according the criteria described in the ARIA guidelines [8] at the screening visits. Peak flow (PEF) measurements were also performed. Due to safety reasons, for all patients, these measurements were performed before and approximately $30 \mathrm{~min}$ after the SCIT injection. If a patient did not demonstrate a PEF of at least $70 \%$ before the injection, the injection was not given and the visit had to be rescheduled. The asthma status of all patients was monitored by the Asthma Control Questionnaire (ACQ) to ensure the control status and identify possible symptoms of undiagnosed asthma. 
Fig. 1. Disposition of patients. ${ }^{1}$ Screening failures were due to not meeting inclusion and/or exclusion criteria, withdrawal of consent, or other reasons; ${ }^{2}$ patient 205/007 was not treated and discontinued already prior to the first dose of IMP; ${ }^{3}$ patients $207 / 003$ and 207/006 were randomized to the standard group but received the 1-strength treatment; ${ }^{4}$ premature discontinuations were due to AEs, withdrawal of consent, or other reasons; ${ }^{5}$ patients were assigned to the SAF as treated; ${ }^{6}$ completed patients are those patients who did not discontinue the trial prematurely. AE, adverse event; APS, All-Patients Set; disc., discontinued; IMP, investigational medicinal product; SAF, Safety Set.

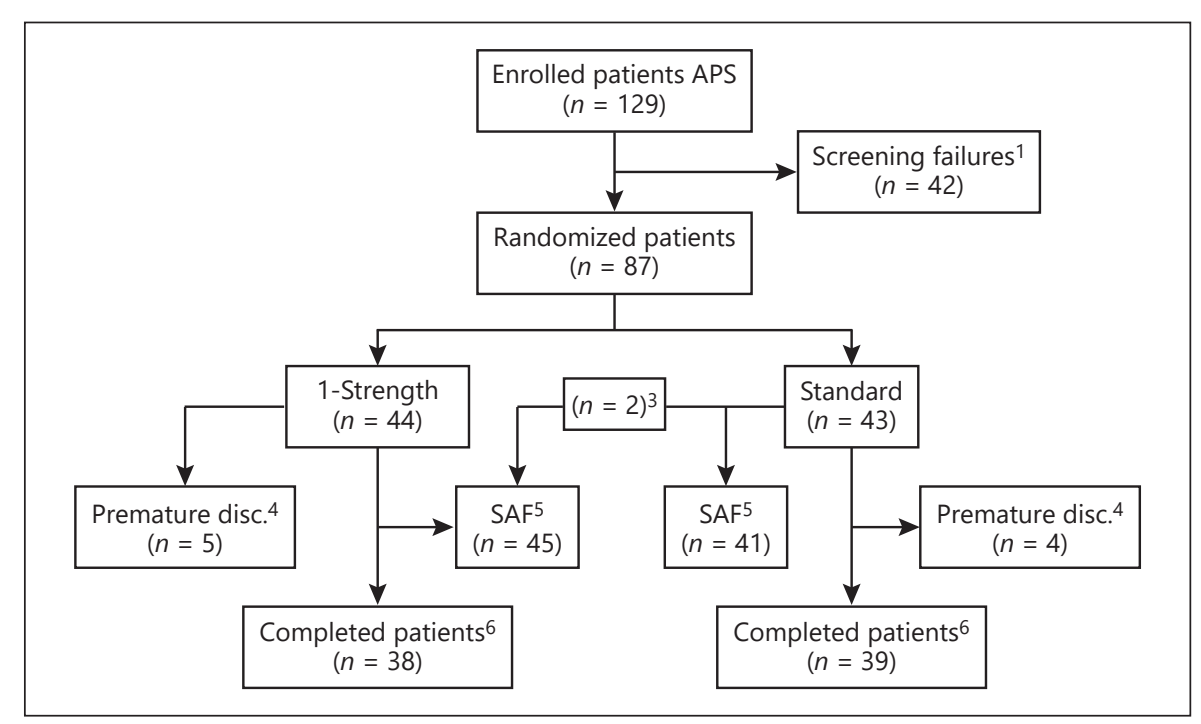

Assessment of Safety and Tolerability End Points

The primary aim of the trial was to obtain information about the safety and tolerability of an accelerated dose escalation schedule for specific immunotherapy with the grass pollen allergoid. The analysis of AEs focused on treatment-emergent AEs (TEAEs), defined as any AE that started or worsened after the first intake of trial medication until 30 days after the last administration of the investigational medicinal product (IMP) or trial-related procedure.

A systemic allergic reaction was defined as an AE related to the IMP and graded according to the WAO grading system based on the organ systems involved and the severity of the reaction.

The following descriptive safety variables were considered: the number, incidence, time of onset, type, and intensity of the AE as well as any serious AE assessed as being drug-related (by the investigator); the incidence and intensity of allergic systemic reactions after injections according to WAO grading; the number of patients reaching the maintenance dose without dose adjustment due to AEs; changes in laboratory values (hematology, clinical chemistry, and urinalysis) measured before and after the treatment phase; changes in vital signs and lung function measured before and after the treatment phase; the assessment of overall tolerability, by the investigator and the patient, using a 5-point Likert scale ( $1=$ very bad; 5 = very good). The trial was supervised by an independent Data and Safety Monitoring Board.

\section{Statistical Analysis}

Due to the exploratory design of the trial, there was no formal estimation of sample size, accounting for the type I error rate, power, $\mathrm{SD}$, and effect size. It was planned to randomize 35 patients per treatment group, to guarantee a probability of $95 \%$ that AEs with a true incidence rate of $8.6 \%$ occur at least once in the treatment group.

The patients were assigned to the following sets before starting the analysis: the "All-Patients Set" (APS) comprised patients that gave their informed consent. The number of these patients equals the total number of screened patients. For this group, demographic data, the patient's disposition, and reasons for premature trial termination are described. The "Safety Set" (SAF) was the group of patients who received at least 1 dose of trial medication. It is the basic analysis set for all assessments of safety and tolerability. For this set, exposure to AIT was analyzed.

Numbers and incidence rates of AEs and severe AEs related to the IMP, and the differences between these numbers and the incidence rates of the 2 treatment groups are reported. For the incidence rates, 95\% confidence intervals (CIs) are also reported. AEs were analyzed separately for the dose escalation phase and the total treatment phase. The results of the tolerability assessments are presented as $n$ (\%) for each category as well as nonparametric statistical measures. Statistical tests for categorical variables (Fisher's exact test, $\chi^{2}$ test, and independent $t$ test) were performed when adequate. Otherwise, the analysis was performed descriptively, and explained by comparing events and frequencies between groups. For all statistical tests, a significance level of $\alpha=5 \%$ was chosen.

\section{Results}

\section{Trial Population}

For this trial, 129 patients were screened, 87 were randomized (1-strength group, $n=44$; standard group, $n=$ $43)$, and 77 completed the trial $(88.5 \%)$. One patient in the 1-strength group dropped out before the first SCIT injection due to flu-like symptoms. Therefore, 86 patients were eligible for the safety analysis. Due to the wrong dose allocation for 2 patients (both were initially randomized to the standard group but received the 1 -strength dose), the safety set comprised 45 patients in the 1-strength group and 41 in the standard group. Nine additional patients terminated the trial prematurely, 5 from the 1-strength group and 4 from the standard group (Fig. 1). Two patients in the 1-strength group discontinued the study because of TEAEs (1 for injection-site erythema and swelling; 1 due to restlessness) versus none in
Kopp/Bovermann/Klimek 
Table 1. Demographic and baseline characteristics of the study population

\begin{tabular}{|c|c|c|c|}
\hline & $\begin{array}{l}\text { Group } 1 \\
(n=45)\end{array}$ & $\begin{array}{l}\text { Group } 2 \\
(n=41)\end{array}$ & $\begin{array}{l}\text { Overall } \\
(n=86)\end{array}$ \\
\hline \multicolumn{4}{|l|}{ Age, years } \\
\hline Mean (SD) & $32.7(9.44)$ & $36.0(12.21)$ & $34.3(10.91)$ \\
\hline Median & 33.0 & 36.0 & 33.0 \\
\hline Range & $18-60$ & $18-65$ & $18-65$ \\
\hline \multicolumn{4}{|l|}{ Gender, $n(\%)$} \\
\hline Male & $27(60.0)$ & $21(51.2)$ & $48(55.8)$ \\
\hline Female & $18(40.0)$ & $20(48.8)$ & $38(44.2)$ \\
\hline \multicolumn{4}{|l|}{ Ethnicity, $n(\%)$} \\
\hline White & $44(97.8)$ & $40(97.6)$ & $84(97.7)$ \\
\hline American Indian or Alaskan Native & $1(2.2)$ & 0 & $1(1.2)$ \\
\hline Asian & 0 & $1(2.4)$ & $1(1.2)$ \\
\hline \multicolumn{4}{|l|}{$\mathrm{BMI}, \mathrm{kg} / \mathrm{m}^{2}$} \\
\hline Mean (SD) & $25.4(4.00)$ & $25.5(6.04)$ & $25.4(5.05)$ \\
\hline Median & 25.0 & 23.9 & 24.7 \\
\hline Range & $19-36$ & $18-44$ & $18-44$ \\
\hline \multicolumn{4}{|l|}{ Smoking status, $n(\%)$} \\
\hline Never & $36(80.0)$ & $31(75.6)$ & $67(77.9)$ \\
\hline Ex-smoker & $6(13.3)$ & $4(9.8)$ & $10(11.6)$ \\
\hline Current smoker & $3(6.7)$ & $6(14.6)$ & $9(10.5)$ \\
\hline \multicolumn{4}{|l|}{ Pet contact, $n(\%)$} \\
\hline No & $35(77.8)$ & $32(78.0)$ & $67(77.9)$ \\
\hline Intermittent & 0 & $2(4.9)$ & $2(2.3)$ \\
\hline Permanent & $10(22.2)$ & $7(17.1)$ & $17(19.8)$ \\
\hline \multicolumn{4}{|l|}{ Total IgE } \\
\hline Median & 150.00 & 224.00 & 166.00 \\
\hline Range & $12.8-1326.0$ & $9.7-3182.0$ & $9.7-3182.0$ \\
\hline \multicolumn{4}{|l|}{ Specific IgE for grass mix/earlybloom } \\
\hline Median & 15.400 & 7.100 & 12.800 \\
\hline Range & $0.36-100.00$ & $1.17-100.00$ & $0.36-100.00$ \\
\hline \multicolumn{4}{|l|}{ Specific $\operatorname{IgG}_{4}$ for Phleum pratense } \\
\hline Median & 0.340 & 0.240 & 0.325 \\
\hline Range & $0.07-8.36$ & $0.07-2.69$ & $0.07-8.36$ \\
\hline
\end{tabular}

BMI, body mass index; Ig, immunoglobulin.

the standard group. Other reasons for premature termination of the trial were the withdrawal of consent and protocol deviation (online suppl. Table S1; see www. karger.com/doi/10.1159/000503684 for all online suppl. material).

The demographic and baseline characteristics were well balanced between the 2 groups (Table 1). Before treatment, all patients experienced nasal symptoms and the majority of patients experienced eye symptoms (1-strength group: $n=35$ [77.8\%] vs. standard group: $n=33$ [80.5\%]). Wheeze, shortness of breath and cough was reported for about one-fifth of patients (range 18.6-22.1\%) and chest tightness for $7.0 \%$. Mean and median duration of symptoms were generally comparable between the groups, ex- cept for chest tightness which had a shorter duration in the 1-strength group (mean 9.7 vs. 26.0 years; median 12.0 vs. 21.0 years). Total IgE, specific IgE against grass pollen, specific $\operatorname{IgG}_{4}$ for $P$. pratense (Table 1) and other specific IgE values (i.e., birch, house dust mite, mugwort) did not differ significantly between groups. In the 1-strength group, $36 / 45$ patients (80.0\%), and in the standard group, $39 / 41$ patients (95.1\%) reached the 1st AIT injection of the maintenance phase without a dose adjustment. Of the patients who did not reach the maintenance phase without a dose adjustment, 3 in the 1-strength group and 2 in the standard group experienced TEAEs that led to AIT reduction. The median treatment duration was 54.8 days in the 1 -strength group and 90.5 days in the standard group. 
Table 2. Overview of TEAEs

\begin{tabular}{|c|c|c|c|c|c|c|}
\hline & \multicolumn{2}{|c|}{ Group $1(n=45)$} & \multicolumn{2}{|c|}{ Group $2(n=41)$} & \multicolumn{2}{|c|}{ Overall $(n=86)$} \\
\hline & $n(\%)$ & $\mathrm{e}$ & $n(\%)$ & $\mathrm{e}$ & $n(\%)$ & $\mathrm{e}$ \\
\hline TEAEs & $37(82.2)$ & 200 & $35(85.4)$ & 244 & $72(83.7)$ & 444 \\
\hline TEAEs related to AIT & $27(60.0)$ & 129 & $20(48.8)$ & 132 & $47(54.7)$ & 261 \\
\hline Local reactions & $26(57.8)$ & 110 & $20(48.8)$ & 123 & $46(53.5)$ & 233 \\
\hline Systemic allergic reactions & $4(8.9)$ & 14 & $1(2.4)$ & 1 & $5(5.8)$ & 15 \\
\hline Other type of events & $3(6.7)$ & 5 & $3(7.3)$ & 8 & $6(7.0)$ & 13 \\
\hline TEAEs leading to discontinuation & $2(4.4)$ & 2 & 0 & 0 & $2(2.3)$ & 2 \\
\hline Treatment-emergent SAE & $1(2.2)$ & 2 & 0 & 0 & $1(1.2)$ & 2 \\
\hline Treatment-emergent SAE related to AIT & $1(2.2)$ & 2 & 0 & 0 & $1(1.2)$ & 2 \\
\hline
\end{tabular}

$n(\%)$ refers to patients who experienced at least 1 TEAE. e, number of events (TEAEs); AIT, allergen immunotherapy; SAE, serious adverse event; TEAE, treatment-emergent adverse event.

\section{Adverse Events}

Overall, 72 (83.7\%) patients reported at least 1 TEAE (Table 2). The proportion of patients with at least 1 TEAE during the trial was similar in both groups (1-strength group: $82.2 \%$; standard group: $85.4 \%$ ). All TEAEs (related or not related to AIT) are summarized in online supplementary Table S2. Most of the reported TEAEs associated with AIT were local reactions at the injection site. There were 233 local reactions with a similar distribution in the 2 groups (110 in the 1-strength group and 123 in the standard group). However, slightly more patients in the 1-strength group experienced at least 1 local reaction (57.8 vs. $48.8 \%)$. Two patients in the 1 -strength group terminated the trial prematurely due to a TEAE. No deaths and no serious unexpected serious adverse reactions were reported during the trial.

All TEAEs assessed by the investigator as being linked to the AIT are summarized in Table 3. Overall, the most commonly reported local reactions were injection-site swelling ( $40.7 \%$ of patients, 87 TEAEs), followed by injection-site erythema (32.6\% of patients, 79 TEAEs), and injection-site pruritus ( $24.4 \%$ of patients, 50 TEAEs). Injection-site pain, injection-site warmth, injection-site hemorrhage, and injection-site urticaria were reported in $\leq 5.8 \%$ of patients.

Of all reported 261 TEAEs related to AIT, most were of mild intensity (230 TEAEs, $88.1 \%$ ) and occurred to a similar extent in both groups. Only 3 TEAEs related to AIT (1.1\%) were classified as severe, and all 3 occurred in patients in the 1-strength group; 2 of them were reported in 1 patient and were also assessed as being serious.

\section{Systemic Adverse Reactions}

Overall, systemic allergic reactions occurred in 5 (5.8\%) patients, $4(8.9 \%)$ in the 1 -strength group and 1 $(2.4 \%)$ in the standard group. However, this difference was not statistically significant $(p=0.3629$, two-sided Fisher's exact test). Of 15 events reported overall, 14 occurred in the 1-strength group. Events occurring more than once in at least 1 patient were rhinorrhea and dyspnea (both in 1 patient) and a decreased forced expiratory volume (3 events in 2 patients). All systemic allergic reactions were assessed as nonserious and no systemic TEAE with a WAO Grade $>2$ was reported. A detailed analysis of the systemic allergic reactions revealed that 1 trial site reported 11 of 14 systemic allergic reactions in the 1 -strength group; this accounts for nearly $80 \%$ of the systemic allergic reactions in this group. These occurred only in 2 patients; one experienced 8 systemic allergic reactions and the other experienced 3 . This result may be explained by a specific trial site effect which is discussed below.

\section{Time to Onset of TEAEs Related to AIT}

No significant difference was observed in the time to onset after AIT administration between the 2 groups. Most of the TEAEs occurred in the first $2 \mathrm{~h}$ (online suppl. Table S3). However, the number of TEAEs in relation to individual AIT injection over the treatment period showed different patterns between the 2 groups. For the 1-strength group, most TEAEs occurred after the first AIT injection, with a continuous decrease in the number of TEAEs along with further injections. In the standard group, the number of AIT-related TEAEs after the first, second, and third injection was low compared to the
Kopp/Bovermann/Klimek 
Table 3. TEAE related to AIT and intensity of TEAEs

\begin{tabular}{|c|c|c|c|c|c|c|}
\hline & \multicolumn{2}{|c|}{ 1-Strength $(n=45)$} & \multicolumn{2}{|c|}{ Standard $(n=41)$} & \multicolumn{2}{|c|}{ Overall $(n=86)$} \\
\hline & $n(\%)$ & $\mathrm{e}$ & $n(\%)$ & $\mathrm{e}$ & $n(\%)$ & $\mathrm{e}$ \\
\hline Mild & & $110(85.3)$ & & $120(90.9)$ & & $230(88.1)$ \\
\hline Moderate & & $16(12.4)$ & & $12(9.1)$ & & $28(10.7)$ \\
\hline Severe & & $3(2.3)$ & & 0 & & $3(1.1)$ \\
\hline At the injection site & $21(46.7)$ & 49 & $14(34.1)$ & 38 & $35(40.7)$ & 87 \\
\hline \multicolumn{7}{|l|}{ Swelling } \\
\hline Erythema & $13(28.9)$ & 28 & $15(36.6)$ & 51 & $28(32.6)$ & 79 \\
\hline Pruritus & $14(31.1)$ & 24 & $7(17.1)$ & 26 & $21(24.4)$ & 50 \\
\hline Pain & $2(4.4)$ & 2 & $3(7.3)$ & 7 & $5(5.8)$ & 9 \\
\hline Warmth & $3(6.7)$ & 5 & 0 & 0 & $3(3.5)$ & 5 \\
\hline Respiratory, thoracic, and mediastinal disorders & $3(6.7)$ & 6 & 0 & 0 & $3(3.5)$ & 6 \\
\hline Nervous system disorders & $1(2.2)$ & 1 & $1(2.4)$ & 1 & $2(2.3)$ & 2 \\
\hline Headache & $1(2.2)$ & 1 & $1(2.4)$ & 1 & $2(2.3)$ & 2 \\
\hline Skin and subcutaneous tissue disorders & $2(4.4)$ & 3 & 0 & 0 & $2(2.3)$ & 3 \\
\hline
\end{tabular}

$n(\%)$ refers to patients who experienced at least one TEAE. e, number of events (TEAEs); AIT allergen immunotherapy; IMP, investigational medicinal product; TEAE, treatment-emergent adverse event.

1-strength group, with the peak of TEAEs occurring after the fourth injection (Fig. 2).

\section{Tolerability and Other Safety Parameters}

Most of the investigators assessed overall tolerability at the end of the escalation phase as "very good" or "good" (68.4 and $26.3 \%$, respectively). Similar results were obtained in the patients' assessment (53.9 and 40.8\%, respectively). The assessment results were similar in both groups (Fig. 3), with neither the statistical tests nor the CIs showing any statistically significant differences. Furthermore, the investigators' and patients' assessments at the final visit revealed similar results. There were also no notable differences between the asthmatic and nonasthmatic patients.

There were no clinically noteworthy changes in mean and median clinical chemistry, hematology, and urinalysis values during the trial; the results were similar in the 2 groups. The immunological profile was assessed at baseline and at the final visit. During the course of the trial, the mean amount of $\operatorname{IgG}_{4}$ against Timothy grass pollen increased notably over time in both groups (Wilcoxon signed-rank test $p<0.0001)$. The comparison of the mean changes from baseline revealed no notable difference between groups at final visit (the van Elteren test: two-sided, $p=0.2552)$.

\section{Discussion}

Allergen-specific immunotherapy is the only treatment that can modify the underlying course of the allergic diseases AR and allergic asthma [3, 4]. However, real-life compliance and adherence to AIT is limited by the longterm updosing and maintenance phases [9]. Recently, Chaker et al. [6] demonstrated that an accelerated 4-dose escalation scheme can be administered with a favorable safety profile that is comparable to the standard 7-dose escalation regime.

Here, we were able to demonstrate that an accelerated high-dose escalation schedule with 1 strength for AIT with a grass pollen allergoid can be used in patients with $\mathrm{AR}$ with or without asthma, and that it has a safety and tolerability profile comparable to the standard escalation schedule. Overall, $82.2 \%$ of patients reported at least 1 TEAE in the high-dose escalation group com- 


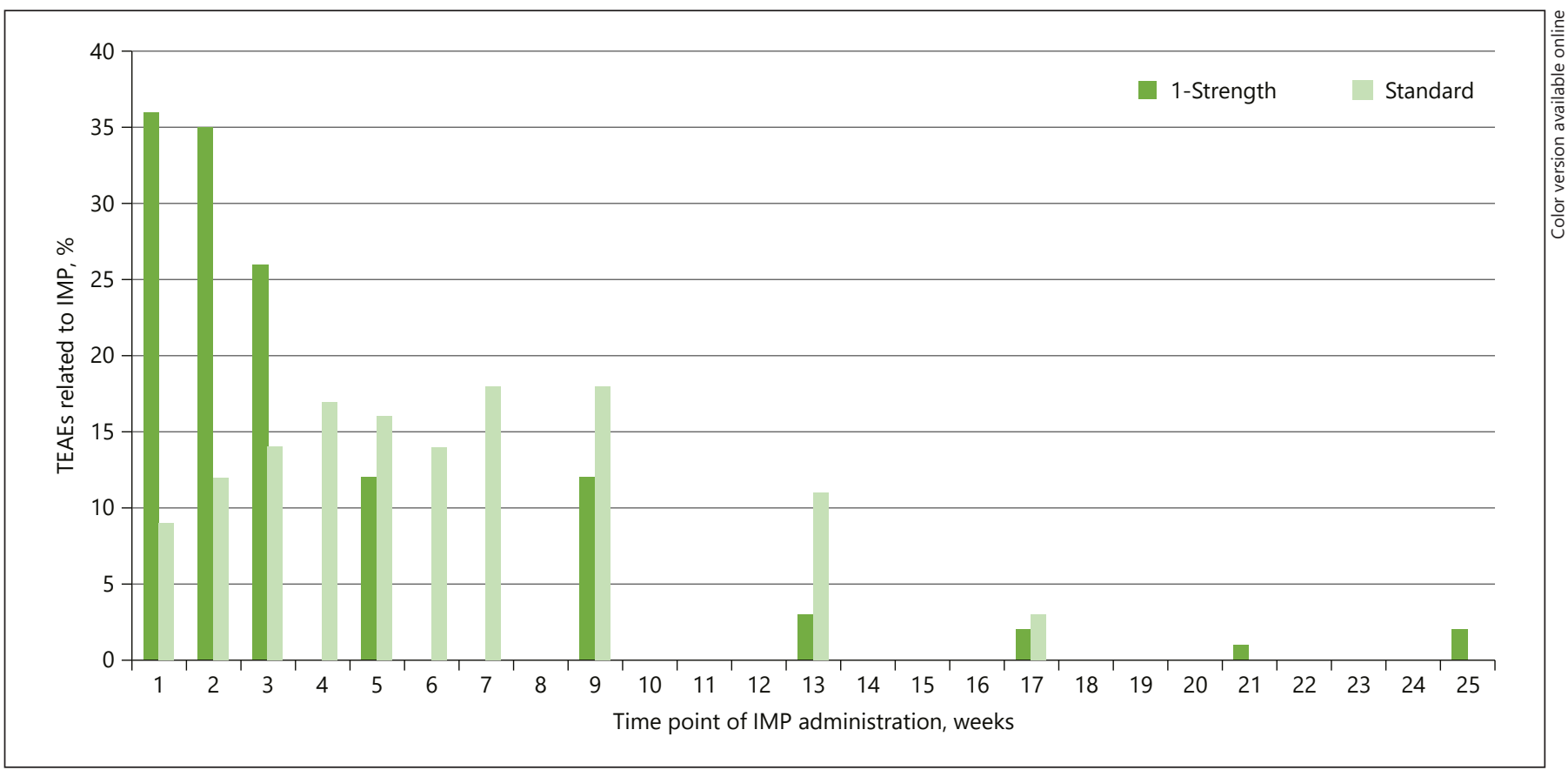

Fig. 2. TEAEs (\%) related to AIT and in relation to individual injections. TEAE, treatment-emergent adverse event.

Fig. 3. Assessment of overall tolerability in the 2 groups by the investigator $(\mathbf{a}, \mathbf{b})$ and the patient $(\mathbf{c}, \mathbf{d})$ after the last dose of the escalation phase on a 5-point Likert scale (very bad - bad - average - good - very good).

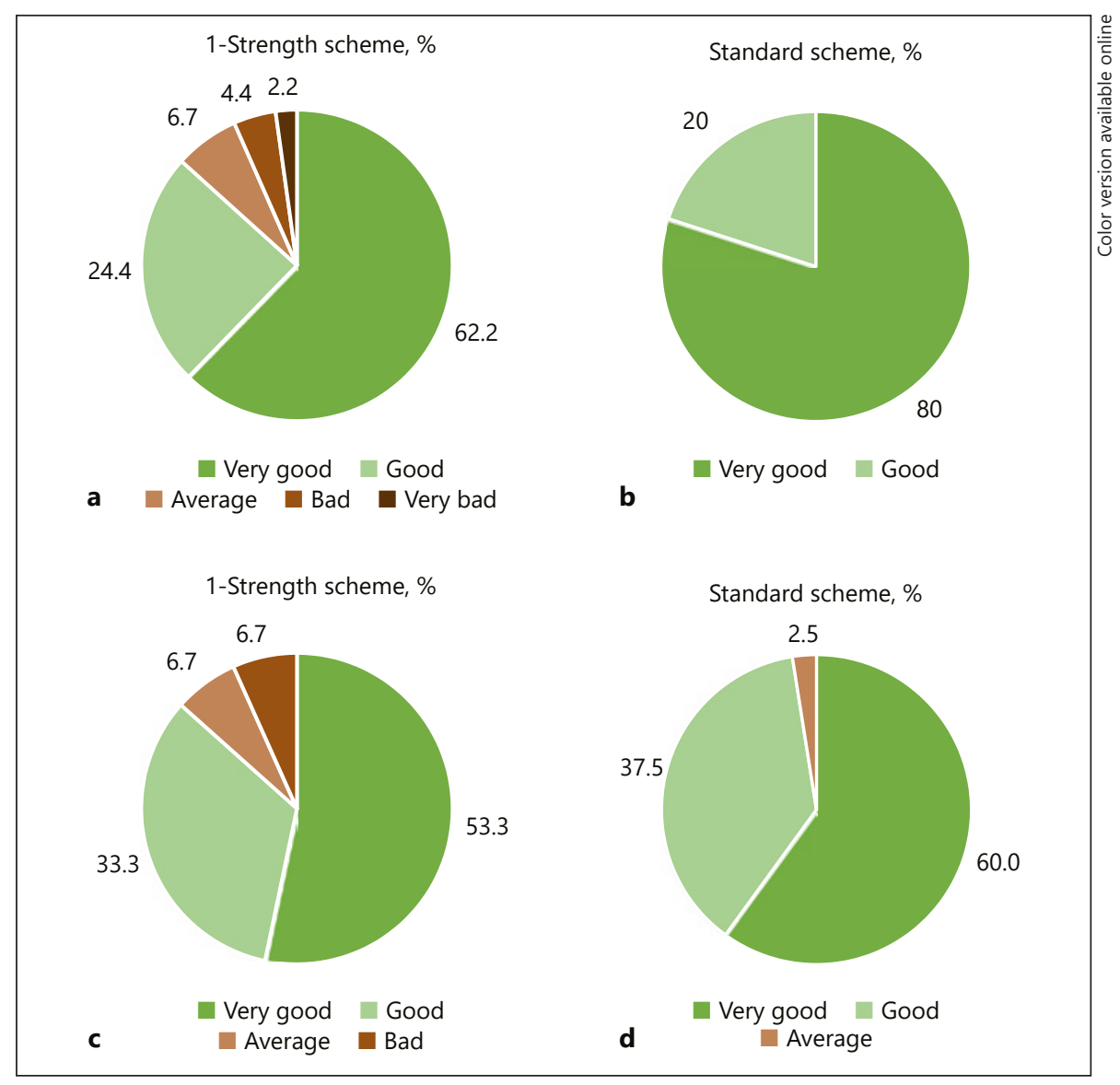


pared to $85.4 \%$ in the standard dose escalation group in this multicenter, open-label, randomized phase II trial. Of note, the majority of patients in the high-dose escalation group (80.0\%) reached the first AIT injection of the maintenance phase without any dose adjustment. Of those patients who did not reach the maintenance phase without dose adjustment, only 3 patients in the highdose escalation group had TEAEs that led to an AIT dose reduction.

A slightly higher number of patients in the high-dose escalation group than in the standard dose escalation group had systemic allergic reactions ( $n=4$ [8.9\%] vs. $n=1[2.4 \%]$ ), but these were all classified as being WAO Grade 1 or 2 . As outlined above, analysis of these events revealed that nearly $80 \%$ of the systemic allergic reactions in the high-dose escalation group were reported in only 2 patients from 1 trial site. We are therefore of the opinion that this result could be explained by a specific trial site effect. This interpretation is supported by the finding that for 1 of these 2 patients, injection-site hematoma was also described, which could indicate an incorrect AIT administration that then led to greater occurrence of systemic allergic reactions. Indeed, specific reevaluation of the administration technique at this study site revealed an incorrect injection technique. In summary, a higher number of systemic allergic reactions does not necessarily indicate an accumulation due to the accelerated dose escalation scheme, but rather points to a specific trial site effect in combination with incorrect AIT handling.

Recently, Tophof et al. [10], in a noninterventional, prospective, observational, longitudinal study, assessed the incidence of local and systemic side effects of SCIT in 581 pediatric patients and 10,015 injections. The incidence of the observed immediate local side effects was as high as in studies on adult patients receiving SCIT [11]. A total of $54.6 \%$ of the patients experienced immediate local side effects at least once; immediate and delayed systemic adverse reactions occurred in 2.2 and $7.4 \%$ of patients, respectively. The number of local and systemic AEs was comparable to the high-dose escalation group in our study population [10].

The time to onset of TEAEs after the AIT injections was similar in both groups in our clinical trial. However, a different pattern was observed with respect to the association of AEs and the individual injections. For example, the highest number of AEs in the high-dose escalation group occurred after the first injection. In contrast, AEs peaked in the standard dose escalation group after the fourth injection. Comparing the cumulative amounts of the AIT products, this peak was observed at a dosage of about 1,000 TU. Hypothetically, these data might point towards faster tolerance induction in the high-dose escalation group. Besides the aspects of safety, tolerability, and adherence to therapy, this would be an additional argument to escalate updosing in AIT. However, this is only a hypothesis derived from clinical observation which must still be confirmed with immunological data.

Recently, Pfaar et al. [12] initiated intraseasonal grasspollen AIT with an 8-day updosing regime and observed significant differences in the immunological response as early as week 3, with an increase in IgE-blocking factor and $\mathrm{IgG}_{4}$ levels. We observed a significant increase in specific $\mathrm{IgG}_{4}$ antibodies against $P$. pratense in both groups. As the final blood sample was drawn at the last trial visit, which took place on different days in the 2 groups (day 50 vs. day 90), no direct comparison can be made based on our data with respect to the kinetics of the $\mathrm{IgG}_{4}$ response.

Slightly more patients in the high-dose escalation group discontinued the trial prematurely (6 vs. 4 ). However, only 3 patients dropped out of the trial due to AEs; 1 reported an AE prior to the first dose, and the other 2 (both in Group I) terminated the trial prematurely due to restlessness and severe injection-site erythema accompanied by severe injection-site swelling, respectively. Obviously, additional data in larger cohorts are necessary for the confirmation of the overall good safety profile in our high-dose escalation group.

In conclusion, our results show that, regardless of dosing schedule, AIT with this grass pollen allergoid was safe and well-tolerated in patients with AR or rhinoconjunctivitis with or without comorbid asthma. TEAEs with the high dose and standard dose escalation were comparable, mild or moderate in intensity, and assessed as nonserious in all but 1 patient.

\section{Acknowledgement}

We thank all the participating medical practices for the recruitment of the patients and for their support of this clinical trial.

\section{Statement of Ethics}

The trial design was approved by the ethic committees of the University of Lübeck. The trial was conducted in accordance with the trial protocol, the International Conference on Harmonization Guideline for Good Clinical Practice, applicable local regulations, and the Declaration of Helsinki. Patients willing to participate in the trial were asked to provide their written informed consent after being given sufficient time to consider participating. 


\section{Disclosure Statement}

M.V.K. received a speaker honorarium or consultant fees from the following companies: ALK-Abelló, Allergopharma, Boehringer-Ingelheim, Chiesi, Glaxo, Infectopharm, Meda, Sanofi-Aventis, Leti Pharma, Novartis, and Vertex. X.B. had nothing to declare. L.K. reported grants and personal fees from Allergopharma, Bionorica, Boehringer Ingelheim, and Roxall, Germany, personal fees from MEDA, Sweden, grants and personal fees from Novartis, Switzerland, and GSK, Great Britain, grants and personal fees from
Lofarma, Italy, grants from Biomay, Austria, and HAL, The Netherlands, grants from LETI, Spain, and Bencard, UK, outside of the submitted work.

\section{Funding Source}

The clinical trial was funded by Allergopharma $\mathrm{GmbH} \&$ Co. KG, Reinbek, Germany.

\section{References}

1 Sih T, Mion O. Allergic rhinitis in the child and associated comorbidities. Pediatr Allergy Immunol. $2010 \mathrm{Feb} ; 21$ (1 Pt 2):e107-13.

2 Cingi C, Gevaert P, Mösges R, Rondon C, Hox $\mathrm{V}$, Rudenko M, et al. Multi-morbidities of allergic rhinitis in adults: European Academy of Allergy and Clinical Immunology Task Force Report. Clin Transl Allergy. 2017 Jun;7:17.

3 Pfaar O, Bachert C, Bufe A, Buhl R, Ebner C, Eng $\mathrm{P}$, et al. Guideline on allergen-specific immunotherapy in IgE-mediated allergic diseases: S2k Guideline of the German Society for Allergology and Clinical Immunology (DGAKI), the Society for Pediatric Allergy and Environmental Medicine (GPA), the Medical Association of German Allergologists (AeDA), the Austrian Society for Allergy and Immunology (ÖGAI), the Swiss Society for Allergy and Immunology (SGAI), the German Society of Dermatology (DDG), the German Society of Oto-Rhino-Laryngology, Head and Neck Surgery (DGHNO-KHC), the German Society of Pediatrics and Adolescent Medicine (DGKJ), the Society for Pediatric Pneumology (GPP), the German Respiratory Society (DGP), the German Association of ENT Surgeons (BV-HNO), the Professional Federation of Paediatricians and Youth Doc- tors (BVKJ), the Federal Association of Pulmonologists (BDP) and the German Dermatologists Association (BVDD). Allergo J Int. 2014;23(8):282-319.

4 Pfaar O, Bonini S, Cardona V, Demoly P, Jakob T, Jutel M, et al.; FASIT group. Perspectives in allergen immunotherapy: 2017 and beyond. Allergy. 2018 Jan;73 Suppl 104:5-23.

5 Corrigan CJ, Kettner J, Doemer C, Cromwell $\mathrm{O}$, Narkus A; Study Group. Efficacy and safety of preseasonal-specific immunotherapy with an aluminium-adsorbed six-grass pollen allergoid. Allergy. 2005 Jun;60(6):801-7.

6 Chaker AM, Al-Kadah B, Luther U, Neumann U, Wagenmann M. An accelerated dose escalation with a grass pollen allergoid is safe and well-tolerated: a randomized open label phase II trial. Clin Transl Allergy. 2016 Feb;6(1):4.

7 Reddel HK, Levy ML; Global Initiative for Asthma Scientific Committee and Dissemination and Implementation Committee. The GINA asthma strategy report: what's new for primary care? NPJ Prim Care Respir Med. 2015 Jul;25(1):15050.

8 Cruz AA, Popov T, Pawankar R, AnnesiMaesano I, Fokkens W, Kemp J, et al.; ARIA Initiative Scientific Committee. Common characteristics of upper and lower airways in rhinitis and asthma: ARIA update, in collaboration with GA(2)LEN. Allergy. 2007;62(s84 Suppl 84):1-41.

9 Kiel MA, Röder E, Gerth van Wijk R, Al MJ, Hop WC, Rutten-van Mölken MP. Real-life compliance and persistence among users of subcutaneous and sublingual allergen immunotherapy. J Allergy Clin Immunol. 2013 Aug;132(2):353-60.e2.

10 Tophof MA, Hermanns A, Adelt T, Eberle P, Gronke C, Friedrichs F, et al. Side effects during subcutaneous immunotherapy in children with allergic diseases. Pediatr Allergy Immunol. 2018 May;29(3):267-74.

11 Calabria CW, Coop CA, Tankersley MS. The LOCAL Study: local reactions do not predict local reactions in allergen immunotherapy. J Allergy Clin Immunol. 2009 Oct;124(4):73944.

12 Pfaar O, Wolf $\mathrm{H}$, Klimek L, Schnitker J, Wüstenberg E. Immunologic effect and tolerability of intra-seasonal subcutaneous immunotherapy with an 8-day up-dosing schedule to 10,000 standardized qualityunits: a double-blind, randomized, placebocontrolled trial. Clin Ther. 2012 Oct;34(10): 2072-81. 